\title{
Ruptured aortic dissection into the left atrium which presented as congestive heart failure and was diagnosed by transoesophageal echocardiography
}

\author{
G Van Camp, I Liebens, P G Silance, B Cham, J L Vandenbossche
}

\begin{abstract}
A 72 year old man was admitted with severe dyspnoea. Ten days before he had had intense thoracic pain with loss of consciousness that was followed by increased dyspnoea. A continuous murmur was heard in the precordial and the left infrascapular regions. Lung auscultation showed stasis over the lower half of both lungs. Transthoracic echocardiography showed a bicuspid aortic valve and a dissection of the proximal aorta, which was considerably enlarged. Transoesophageal echocardiography confirmed dissection of the proximal aorta and showed a communication from the false lumen of the aortic dissection to the left atrium; and colour flow Doppler showed a continuous shunt to the left atrium. After transoesophageal echocardiography the patient had emergency surgical repair, which was successful. He had no complications in the postoperative period.
\end{abstract}

(Br Heart f 1994;72:400-402)

Transoesophageal echocardiography is the best method of diagnosing aortic dissection of the thoracic aorta in the emergency room. ${ }^{12}$ Ruptured aortic dissection into a cardiac chamber is rare. Cases of rupture into the right atrium have been described; but rupture into the left atrium has been described only as an incidental finding at necropsy and in a patient in whom an intimal flap in the left atrium was found by transthoracic cross sectional echocardiography. ${ }^{34}$ This patient died soon after angiography and before surgery could be performed.

We describe a case of ruptured aortic dissection into the left atrium. This patient presented with the clinical signs typical of this disease and all the details needed for early successful operation were supplied by transoesophageal echocardiography.

Case report

A 70 year old man was admitted to our hospi- tal with increasing dyspnoea. He had a history of chronic obstructive lung disease but not of systemic arterial hypertension or other cardiovascular disease. There was no family history of cardiovascular disease. Ten days before admission he had had intense chest pain and lost consciousness. The chest pain persisted for 2 hours and since then the dyspnoea had progressively increased. On admission the patient had polypnoea, he was slightly cyanosed, and had a normal blood pressure (120/80 $\mathrm{mm} \mathrm{Hg}$ for both arms).

Auscultation showed a diamond shaped systolic murmur $3 / 6$ in the second right intercostal region and a continuous murmur at the apex and in the left infrascapular area. There was stasis in the lower half of both lungs. He was slightly disorientated but had no other neurological abnormality.

A 12 lead electrocardiogram showed left atrial hypertrophy. Chest $x$ ray examination showed a slightly enlarged cardiac silhouette, pulmonary congestion, and equivocal evidence of aortic ectasia. Measurement of blood gases showed hypoxaemia: $\mathrm{pH} 7 \cdot 47$, $\mathrm{pO}_{2} 68 \mathrm{~mm} \mathrm{Hg}, \mathrm{pCO}_{2} 33 \mathrm{~mm} \mathrm{Hg}$.

Cross sectional transthoracic echocardiography showed a bicuspid calcified aortic valve with a reduced orifice and an enlarged proximal aorta $(70 \mathrm{~mm})$ which seemed to contain an intimal flap. Doppler echocardiography showed a maximum gradient of 30 $\mathrm{mm} \mathrm{Hg}$ across the aortic valve. No aortic regurgitation was detected by Doppler examination.

Transoesophageal echocardiography confirmed the aortic dissection, which was limited to the proximal aorta. The intimal tear started just above the origin of the coronary arteries. There was a clear discontinuity between the false lumen of the aortic dissection and the left atrium (fig 1) and colour Doppler showed a continuous flow from the false lumen to the left atrium (fig 2). There was a small pericardial effusion. The surgical team was informed and the patient had immediate surgical repair.

The surgeon confirmed the presence of a significantly enlarged proximal aorta with a large false lumen that communicated with the left atrium. There was no other sign of 


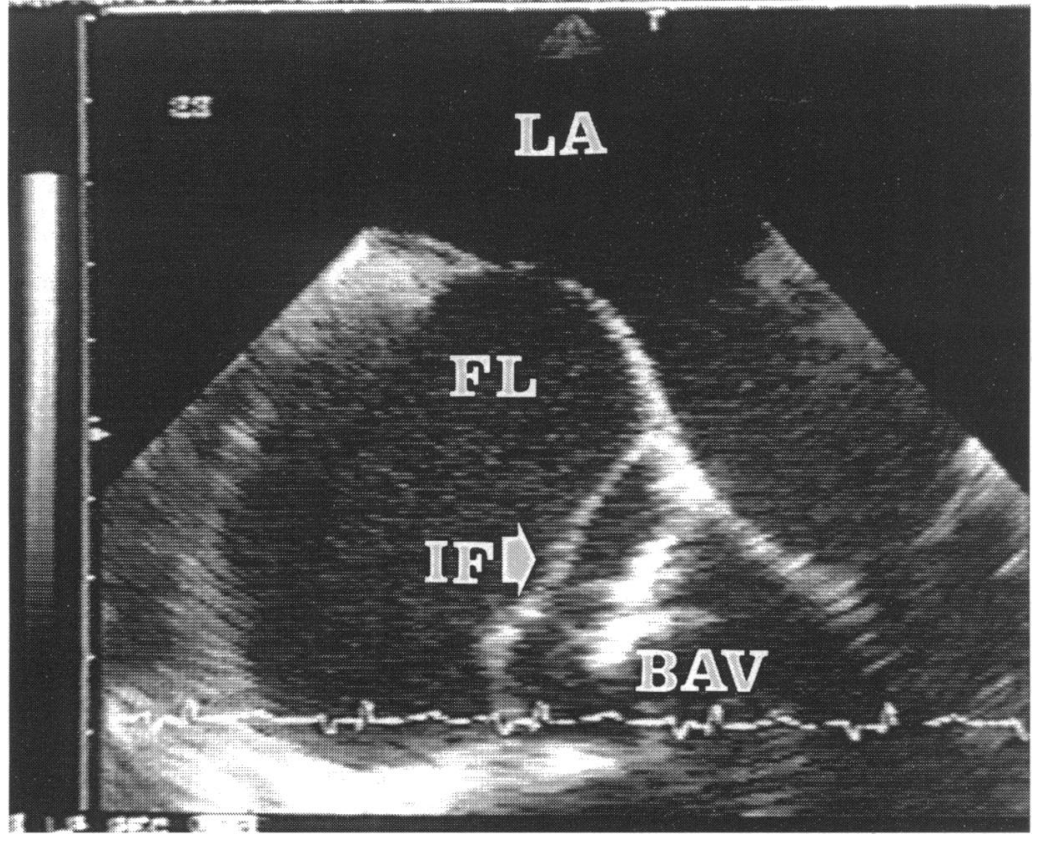

Figure 1 Transverse transoesophageal echocardiographic view showing aortic dissection of the proximal aorta and discontinuity (arrow) between the false lumen of the aortic dissection and the left atrium ( $L A$, left atrium; $B A V$, bicuspid aortic valve; IF, intimal flap; FL, false lumen). uncomplicated recovery. A follow up examination by transoesophageal echocardiography showed no residual dissection and no communication between the aorta and the heart chambers.

\section{Discussion}

This patient had no history of systemic arterial hypertension and he had no signs of Marfan's syndrome. Transoesophageal echocardiography showed an almost normal descending aorta without atherosclerotic plaques. An uncommon association between aortic dissection caused by cystic media necrosis and bicuspid aortic valves has been described in patients without the overt clinical signs of Marfan's syndrome. ${ }^{6}$ Though we cannot rule out a form fruste of Marfan's syndrome in our patient, we think it more likely that cystic media necrosis caused the aortic dissection.

The clinical features in our patient are compatible with a ruptured aneurysm of the sinus of Valsalva into the left atrium - that is, congestive heart failure with a continuous murmur in the left infrascapular area. The intense pain preceding the symptoms of congestive heart failure, however, were clearly caused by dissection of the proximal aorta. Transoesophageal echocardiography showed the bicuspid aortic valve without significant stenosis, no involvement of the coronary arteries, an intimal tear just above the origin of the coronary arteries, a communication

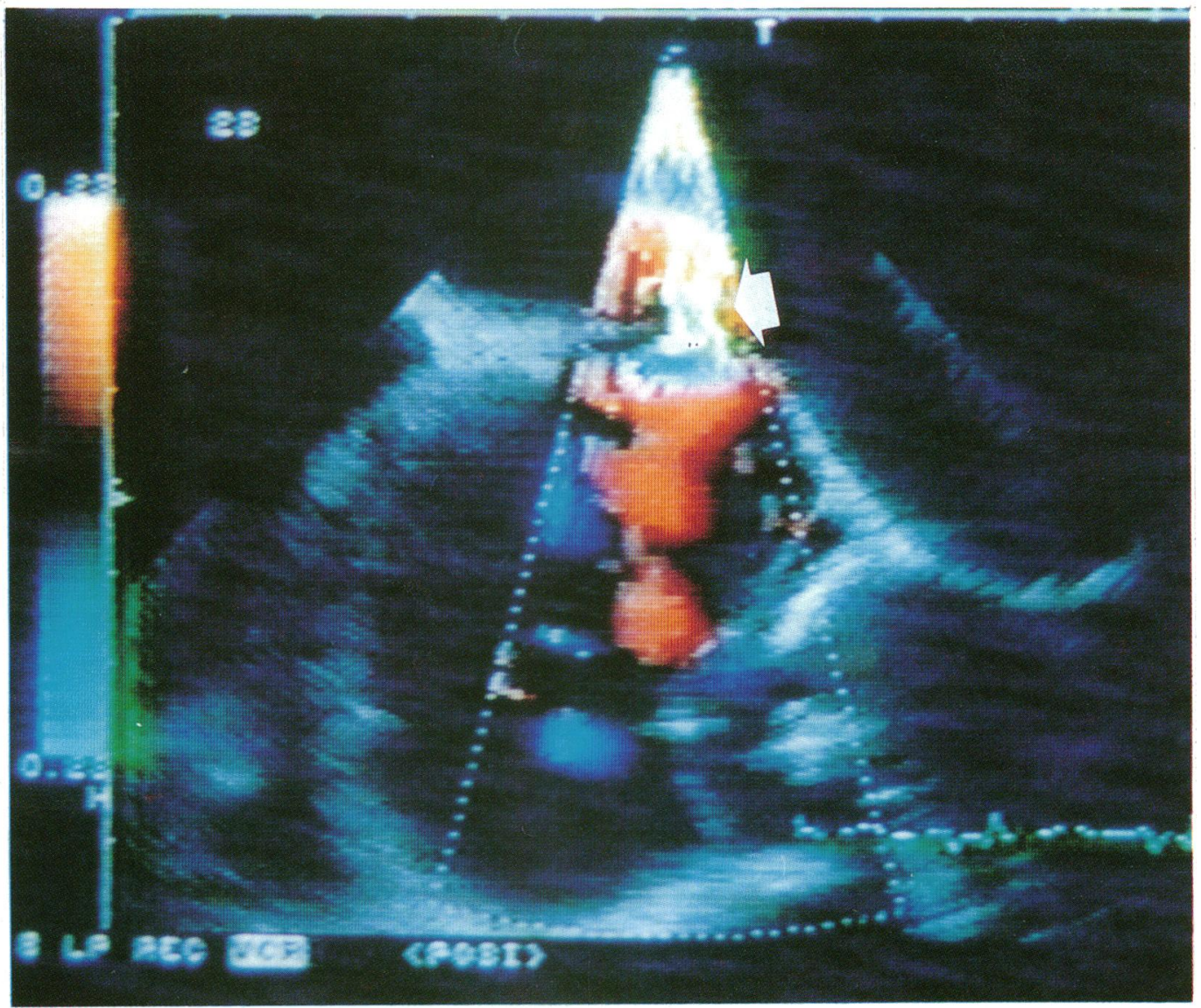

Figure 2 Transverse transoesophageal echocardiographic view showing continuous flow from the false lumen of the aortic dissection to the left atrium (arrow). 
between the false lumen of the aortic dissection and the left atrium, and an aortic dissection limited to the proximal aorta. This information led to immediate and successful surgical repair.

1 Borner N, Erbel R, Braun B. Diagnosis of aortic dissection by transoesophageal echocardiography. Am $\mathcal{F}$

2 Erbel R, Engberdmy W, Daniel W. Echocardiography in diagnosis of aortic dissection. Lancet 1989;i:457-61.
3 Boyd LJ. A study of thousand reported cases of aneurysm of the thoracic aorta. Am $\mathcal{F}$ Med Sci 1924;168:654-68.

4 Hurley DV, Nishimura RA, Shaff HV, Edwards WD. Aortic dissection with fistula to right atrium. A noninvasive diagnosis by two-dimensional and Doppler echocardiography with successful repair. $\mathcal{F}$ Thorac Cardiovasc Surg 1986;92:953-7.

5 Oliveira JS, Basteti RB, Marin-Neto JA, Silva Costa R, Carneiro JJ. Ruptured aortic dissection into the left atrium: a rare cause of congestive heart failure. $\mathrm{Am}$ Heart $f$ 1991;21:936-8.

6 Mac Kusick VA, Logue RB, Bahnson HT. Association of aortic valvular disease and cystic medial necrosis of the ascending aorta; report of four instances. Circulation 1957;16:188-90. 